\title{
A Robust Joint Optimal Pricing and Lot-Sizing Model
}

\author{
Sungmook Lim* \\ Division of Business Administration, Korea University
}

(Received: July 27, 2012 / Revised: August 22, 2012 / Accepted: August 24, 2012)

\begin{abstract}
The problem of jointly determining a robust optimal bundle of price and order quantity for a retailer in a singleretailer, single supplier, single-product supply chain is considered. Demand is modeled as a decreasing power function of product price, and unit purchasing cost is modeled as a decreasing power function of order quantity and demand. Parameters defining the two power functions are uncertain but their possible values are characterized by ellipsoids. We extend a previous study in two ways; the purchasing cost function is generalized to take into account the economies of scale realized by higher product demand in addition to larger order quantity, and an exact transformation into an equivalent convex optimization program is developed instead of a geometric programming approximation scheme proposed in the previous study.
\end{abstract}

Keywords: Robust Optimization, Pricing, Lot-Sizing, Convex Optimization, Geometric Program

* Corresponding Author, E-mail: sungmook@korea.ac.kr

\section{INTRODUCTION}

The purpose of this short note is to revisit and extend the work of Lim (2008) which proposes a robust pricing and lot-sizing model and a solution method based on geometric programming (GP) approximation. We extend his work in two ways; the purchasing cost function is generalized to take into account the effect of economies of scale realized by higher product demand in addition to larger order quantity, and an exact transformation of the model into an equivalent convex optimization program is developed instead of the GP approximation scheme.

We consider a two-stage supply chain that consists of a single-retailer and a single-supplier in which the retailer seeks to maximize its own profit by determining an optimal order quantity and unit selling price of a single product. The product is assumed to be commoditylike so that its demand is stable (but not constant) and can be boosted by lowering its unit selling price; the lower the price, the higher the demand. This consumption-side relationship can be modeled by describing demand for the product as a decreasing power function of unit selling price. It is also assumed that the supplier offers continuous all-unit quantity discounts schedule to the retailer; the larger the order quantity, the lower the unit purchasing cost. Furthermore, higher demand contributes to the economies of scale in production and helps reduce the manufacturer's production costs. This in turn effectively reduces the retailer's purchasing cost. Considering these two effects, unit purchasing cost can be modeled as a decreasing power function of both order quantity and product demand. Note that Lim (2008) does not consider the effect of product demand on unit purchasing cost.

There have been many studies which investigated the problem of jointly determining an optimal bundle of price and order quantity described above. For some of those, we refer the reader to Abad (1988, 1996, 2003), Dye (2007), Goh and Moosa (2002), Jørgensen and Kort (2002), and Viswanathan and Wang (2003). However, to the best of our knowledge, there are few studies which deal with uncertainties involved in the model's parameters. This is in part due to the fact that EOQ-type lot-sizing models are very insensitive to the variation of 
parameters (Yu, 1997). While this is true for parameters, such as ordering cost, inventory carrying cost rate, which are coefficients of linearly added cost terms, any uncertainties in the exponents of the demand and unit purchasing cost functions can have a significant impact on the model's optimal solution. This necessitates a development of relevant models like one in a recent study of Lim (2008), and we intend to extend his work.

This short note is organized as follows. We first introduce our model in Section 2, followed by its transformation into an equivalent convex program in Section 3 . Section 4 concludes the paper by providing some remarks.

\section{MODEL}

The retailer's profit $(\pi(P, Q))$ per unit time is defined as revenue minus ordering cost, inventory holding cost, and purchasing cost:

$$
\pi(P, Q)=P D-s \frac{D}{Q}-i c \frac{Q}{2}-c D
$$

where $P$ and $Q$ are the decision variables representing unit selling price and order quantity, respectively, $s$ is ordering cost, $i$ is inventory carrying cost rate per unit time, $c$ is unit purchasing cost, and $D$ is demand for the product per unit time.

Product demand is assumed to be a decreasing function of product price with constant elasticity; i.e., $D=$ $k P^{-\alpha}$, where $k>0$ and $\alpha>0$ are parameters. Unit purchasing cost is also assumed to be a decreasing function of both demand and order quantity; i.e., $c=r Q^{-\beta} D^{-\mu}$, where $r>0, \beta>0$ and $\mu>0$ are parameters. Since $D=k P^{-\alpha}$, the unit purchasing cost function can be rewritten as $c=r Q^{-\beta} k^{-\mu} P^{\mu \alpha}=w Q^{-\beta} P^{\gamma}$, where $w>0, \beta>0$ and $\gamma>0$ are parameters. Although a certain choice of parameter values may make the problem of maximizing the retailer's profit unbounded (see Lee (1993)), such a choice is assumed to be avoided. Except for the assumption of constant demand and constant unit cost, the same assumptions of the classical economic order quantity (EOQ) model are made: instantaneous replenishment, no stock-outs, and a fixed ordering cost.

By incorporating the demand function and the purchasing cost function into (1), we get the following unconstrained optimization problem for the retailer who seeks to maximize its profit:

$$
\begin{aligned}
\max _{P, Q} \pi= & k P^{-\alpha+1}-s k P^{-\alpha} Q^{-1}-0.5 i w Q^{-\beta+1} P^{\gamma} \\
& -k w P^{-\alpha+\gamma} Q^{-\beta}
\end{aligned}
$$

Because of its negative coefficients in the objective, this optimization problem is in its current form a signomial program, which is nonconvex and may require an exhaustive search to secure a globally optimal solution.
However, since the signomial function in (2) involves only one positive coefficient, it can be transformed into a geometric program assuming all the variables are positive as follows (Duffin et al., 1967):

$$
\begin{aligned}
& \min _{\pi, P, Q} \pi^{-1} \\
& \text { s.t } s P^{-1} Q^{-1}+0.5 i w k^{-1} P^{\alpha+\gamma-1} Q^{-\beta+1} \\
& \\
& \quad+w P^{\gamma-1} Q^{-\beta}+k^{-1} \pi P^{\alpha-1} \leq 1
\end{aligned}
$$

Note that model (3), via a transformation into a convex program, can be solved to global optimality efficiently and reliably using interior-point methods (Boyd and Vandenberghe, 2004).

We now proceed to incorporate an uncertainty of the parameters $k, \alpha, w$ and $\gamma$ defining the demand and purchasing cost functions into model (3). Note that we assume that the other parameters, such as $s, i, \beta$, have certain values. In particular, it is not unrealistic to assume that the value of $\beta$ is certain since it is usually dictated by the quantity discounts offered by the supplier. We further assume that the uncertainty of the parameters can be characterized by ellipsoids as follows:

$$
\begin{aligned}
& \left(\begin{array}{c}
\log k \\
\alpha
\end{array}\right) \in F=\left\{\left(\begin{array}{c}
\overline{\log k} \\
\bar{\alpha}
\end{array}\right)+M v:\|v\|_{2} \leq 1, \quad v \in \Re^{2}\right\} \\
& \left.\left(\begin{array}{c}
\log w \\
\gamma
\end{array}\right) \in G=\left\{\begin{array}{c}
\overline{\log w} \\
\bar{\gamma}
\end{array}\right)+H u:\|u\|_{2} \leq 1, \quad u \in \Re^{2}\right\}
\end{aligned}
$$

where $M=\Re^{2 \times 2}$ and $H \in \Re^{2 \times 2}$ are real symmetric positive definite matrices, and $\left(\begin{array}{c}\overline{\log k} \\ \bar{\alpha}\end{array}\right)$ and $\left(\begin{array}{c}\overline{\log w} \\ \bar{\gamma}\end{array}\right)$ are constant vectors defining the centers of the ellipsoids, $F$ and $G$, respectively. A justification of ellipsoidal representation of the parameter uncertainties will be given in the next section. Then, a (worst-case) robust optimization version of the problem can be formulated as follows:

$$
\begin{aligned}
& \min _{\pi, P, Q} \pi^{-1} \\
& \text { s.t. } s P^{-1} Q^{-1}+\max _{\substack{(\log k, \alpha) \in F \\
(\log w, \gamma) \in G}}\left(k^{-1} \pi P^{\alpha-1}\right. \\
& \\
& \quad+0.5 i w k^{-1} P^{\alpha+\gamma-1} Q^{-\beta+1} \\
& \left.\quad+w P^{\gamma-1} Q^{-\beta}\right) \leq 1
\end{aligned}
$$

Note that it is implicitly assumed that the decision variables take on positive values. Model (6) is the problem of our interest and, to make it tractable, a special treatment is needed, the details of which will be presented in the next section.

\section{ESTIMATION OF PARAMETERS AND THEIR UNCERTAINTIES}

Although it is a generally accepted approach to use 
power functions for modeling the demand and purchasing cost functions under continuous quantity discounts and price-sensitive product demand, it is not practically simple how to obtain the values of these functions' parameters. A convenient approach for getting the parameter values is to run a regression analysis on past sales $(D$ and $P)$ and production data $(Q, P$ and $c)$ and obtain leastsquares estimates. Two monomial fittings are required; one is for the demand function $D=k P^{-\alpha}$ and the other is for the purchasing cost function $c=w Q^{-\beta} P^{\gamma}$. Monomial fitting can be done via linear regression after taking logarithm on the underlying function. Suppose we are given price-demand data pairs $\left(P^{(i)}, D^{(i)}\right), i=1, \cdots, n$. The goal is to fit the data with a monomial $D=k P^{-\alpha}$. It is needed to find $k>0$ and $\alpha$ so that $k\left(P^{(i)}\right)^{-\alpha} \approx D^{(i)}, i=1$, $\cdots, n$. Let $p^{(i)}=\log P^{(i)}, d^{(i)}=\log D^{(i)}$ and replace $k\left(P^{(i)}\right)^{-\alpha}$ $\approx D^{(i)}$ with $\log k\left(e^{p^{(i)}}\right)^{-\alpha} \approx d^{(i)}$, to get $\log k-\alpha p^{(i)} \approx d^{(i)}$, $i=1, \cdots, n$. We can use simple linear regression to find estimates for $\log k$ (intercept) and $\alpha$ (slope) based on least squares given the data $\left(p^{(i)}, d^{(i)}\right), i=1, \cdots, n$. The same procedure can be undertaken to get estimates for $\log w$ and $\gamma$.

While regression estimates for the parameters are certain values for a particular sample data set, these values are subject to sampling errors. In other words, regression estimators are statistics and thus random variables; different estimates can be obtained from different sample data sets. Since small variations in the values of parameters such as price elasticity can have a significant impact on model solution, it is needed to take into account any statistical errors of regression estimates which may be caused by model misfit, unconsidered factors, observation errors, and so on. This consideration necessitates the development of a way of incorporating parameter uncertainty into the model.

It is well known that the least-squares estimators for intercept and slope in a simple linear regression are jointly normally distributed random variables and there exists a non-positive correlation between them, when the sample size is sufficiently large (See Lim (2008) and statistics texts for a more detail). For a pair of two jointly normally distributed random variables, it is quite natural to represent their uncertainty with ellipsoids. When we plot lots of randomly generated incidences of the two random variables, it can be easily observed that an ellipsoidal shape is formed. For a more discussion of ellipsoidal representation of parameter uncertainty, refer to Lim (2007).

Now take a look at the ellipsoids $F$ and $G$, given in (4) and (5). The constant vectors, $\left(\begin{array}{c}\overline{\log k} \\ \bar{\alpha}\end{array}\right)$ and $\left(\begin{array}{c}\overline{\log w} \\ \bar{\gamma}\end{array}\right)$, can be replaced by regression estimates for the corresponding parameters obtained from a particular sample data set. These two constant vectors determine the central locations of the two ellipsoids, and any functional dependency between $k$ and $w(\alpha$ and $\gamma)$ is taken into account by this. On the other hand, $M v$ and $H u$ represent any statistical errors of regression estimates incurred in the two (independent) monomial fittings, respectively. Therefore, within the two ellipsoids $F$ and $G$, the two parameter vectors $\left(\begin{array}{c}\log k \\ \alpha\end{array}\right)$ and $\left(\begin{array}{c}\log w \\ \gamma\end{array}\right)$ can be considered to vary independently with each other. This consideration provides a logical support for the main results presented in the subsequent section.

\section{MAIN RESULTS}

In this section we show that model (6) can be transformed into an equivalent convex optimization program. First let us examine the maximization part in the constraint of model (6), which can be rewritten as follows:

$$
\max _{\substack{(\log k, \alpha) \in F \\(\log w, \gamma) \in G}} k^{-1} P^{\alpha-1}\left(\pi+t w P^{\gamma}\right)+z w P^{\gamma}
$$

where $t:=0.5 i Q^{-\beta+1}$ and $z:=P^{-1} Q^{-\beta}$ are constants. Letting $f^{*}(P):=\max _{(\log k, \alpha) \in F} k^{-1} P^{\alpha-1}$ and $g^{*}(P):=\max _{(\log w, \gamma)}$ ${ }_{\epsilon G} w P^{\gamma}$, we get the following lemma adapted from Lim (2008).

$$
\begin{aligned}
& \text { Lemma1: } f^{*}(P)=e^{(-1 \log P)\left(\frac{\overline{\log k}}{\bar{\alpha}}\right)+\left\|M^{T}\left(\begin{array}{c}
-1 \\
\log P
\end{array}\right)\right\|_{2}^{-\log P}} \text { and } g^{*}(P) \\
& =e^{(1 \log P)\left(\begin{array}{c}
\log w \\
\bar{\gamma}
\end{array}\right)+H^{T}\left(\begin{array}{cc}
1 \\
\log P
\end{array}\right) \|_{2}} \text {. }
\end{aligned}
$$

Proof: By applying logarithm, $\log \left(f^{*}(P)\right)$ can be determined by solving the following equivalent problem:

$$
\begin{gathered}
\log \left(f^{*}(P)\right)=\max _{v}(\alpha-1) \log P-\log k \\
\text { s.t. }\left(\begin{array}{c}
\log k \\
\alpha
\end{array}\right)=\left(\begin{array}{c}
\overline{\log k} \\
\bar{\alpha}
\end{array}\right)+M\left(\begin{array}{c}
v_{1} \\
v_{2}
\end{array}\right) \\
\|v\|_{2} \leq 1
\end{gathered}
$$

Substituting the equality constraint into the objective function, we get

$$
\begin{aligned}
& \log \left(f^{*}(P)\right)=\max _{v}(-1\log P)\left(\begin{array}{c}
\overline{\log k} \\
\bar{\alpha}
\end{array}\right) \\
&+(-1 \log P) M\left(\begin{array}{l}
v_{1} \\
v_{2}
\end{array}\right)-\log P \\
& \text { s.t. }\|v\|_{2} \leq 1
\end{aligned}
$$

the optimal value of which is

$$
\log \left(f^{*}(P)\right)=\left(\begin{array}{ll}
-1 & \log P
\end{array}\right)\left(\begin{array}{c}
\overline{\log k} \\
\bar{\alpha}
\end{array}\right)+\left\|M^{T}\left(\begin{array}{c}
-1 \\
\log P
\end{array}\right)\right\|_{2}-\log P,
$$

hence the result. The case for $g^{*}(P)$ can be derived in 
the same way.

Observe that the maximizations of $k^{-1} P^{\alpha-1}$ and $w P^{\gamma}$ in (7) can be done independently due to the fact that $F$ and $G$ are independent as claimed in the previous section. Therefore, model (6) reduces to the following model (8) due to Lemma 1.

$$
\begin{gathered}
\min _{\pi, P, Q} \pi^{-1} \\
\text { s.t. } \quad s P^{-1} Q^{-1}+e^{\log \left(f^{*}(P)\right)}\left(\pi+0.5 i Q^{-\beta+1} e^{\log \left(g^{*}(P)\right)}\right) \\
+ \\
+P^{-1} Q^{-\beta} e^{\log \left(g^{*}(P)\right)} \leq 1
\end{gathered}
$$

Proposition 1: Model (8) can be transformed into an equivalent convex optimization program.

Proof: First $e^{\log \left(f^{*}(P)\right)}$ and $e^{\log \left(g^{*}(P)\right)}$ can be rewritten as

$$
\begin{aligned}
& e^{\log \left(f^{*}(P)\right)}=e^{(-1 \log P)\left(\begin{array}{c}
(\overline{\log k}) \\
\bar{\alpha}
\end{array}\right)+\left\|M^{T}\left(\begin{array}{c}
-1 \\
\log P
\end{array}\right)\right\|_{2}-\log P} \\
& =e^{-\overline{\log k+\bar{\alpha} \log P+}\left\|M^{T}\left(\begin{array}{c}
-1 \\
\log P
\end{array}\right)\right\|_{2}^{-\log P}} \\
& =\bar{k}^{-1} P^{\bar{\alpha}-1} e^{\left\|M^{T}\left(\begin{array}{c}
-1 \\
\log P
\end{array}\right)\right\|_{2}} \\
& e^{\log \left(g^{*}(P)\right)}=e^{(1 \log P)}\left(\begin{array}{c}
\overline{\log w} \\
\bar{\gamma}
\end{array}\right)+\left\|H^{T}\left(\begin{array}{c}
1 \\
\log P
\end{array}\right)\right\|_{2} \\
& =e^{\overline{\log w}+\bar{\gamma} \log P+\left\|H^{T}\left(\begin{array}{c}
1 \\
\log P
\end{array}\right)\right\|_{2}} \\
& =\bar{w} P^{\bar{\gamma}} e^{\left\|H^{T}\left(\begin{array}{c}
1 \\
\log P
\end{array}\right)\right\|_{2}}
\end{aligned}
$$

where $\bar{k}^{-1}=e^{-\overline{\log k}}$ and $\bar{w}=e^{\overline{\log w}}$. By introducing two new variables, $x$ and $y$, for replacing $e^{\left\|M^{T}\left(\begin{array}{c}-1 \\ \log P\end{array}\right)\right\|_{2}}$ and $e^{\left\|H^{T}\left(\begin{array}{c}1 \\ \log P\end{array}\right)\right\|_{2}}$, respectively, in the objective, we get the following model (9) which is equivalent to model (8):

$$
\begin{aligned}
\min _{\pi, P, Q} \pi^{-1} & \\
\text { s.t. } s P^{-1} Q^{-1} & +\bar{k}^{-1} \pi P^{\bar{\alpha}-1} x \\
& +0.5 i \bar{w} \bar{k}^{-1} P^{\bar{\alpha}+\bar{\gamma}-1} Q^{-\beta+1} x y \\
& +\bar{w} P^{\bar{\gamma}-1} Q^{-\beta} y \leq 1
\end{aligned}
$$

By change of variables, $p:=\log P, q:=\log Q, \phi:=$ $\log \pi, \chi:=\log x, \psi:=\log y$, model (9) is transformed into model (10):

$$
\begin{aligned}
& \min _{p, q, \phi, \chi, \psi}-\phi \\
& \text { s.t. } \quad \log \left(e^{-p-q+\log s}+e^{(\bar{\alpha}-1) p+\phi+\chi+\log \bar{k}^{-1}}\right. \\
& \quad+e^{(\bar{\alpha}+\bar{\gamma}-1) p+(-\beta+1) q+\chi+\psi+\log \left(0.5 i \bar{w} \bar{k}^{-1}\right)}
\end{aligned}
$$

$$
\begin{aligned}
& \left.\quad+e^{(\bar{\gamma}-1) p-\beta q+\psi+\log \bar{w}}\right) \leq 0 \\
& \left\|M^{T}\left(\begin{array}{c}
-1 \\
p
\end{array}\right)\right\|_{2} \leq \chi \\
& \left\|H^{T}\left(\begin{array}{l}
1 \\
q
\end{array}\right)\right\|_{2} \leq \psi
\end{aligned}
$$

The first inequality in (10) is a so-called log-sumexp function which is known to be convex and the second and third constraints are second-order cone constraints which are also known to be convex (see Boyd and Vandenberghe (2004)), hence the result.

\section{CONCLUDING REMARKS}

We have investigated the problem of jointly determining a robust optimal bundle of price and order quantity for the retailer with significant uncertainties involved in the parameters defining the demand and purchasing cost functions. We have shown that the problem can be transformed into an equivalent convex optimization program, which can be efficiently and reliably solved to global optimality using interior-point methods. High-quality convex optimization software such as cvx (Grant and Boyd, 2010) is available, and it is a fairly easy task to solve model (10). As a short note, this paper lacks an extensive computational experiment to investigate the model's behavior and leaves it for a possible further research topic.

\section{ACKNOWLEDGEMENT}

This research was supported by Basic Science Research Program through the National Research Foundation of Korea (NRF) funded by the Ministry of Education, Science and Technology (No. 2012-0002735).

\section{REFERENCES}

Abad, P., "Optimal Pricing and Lot-Sizing under Conditions of Perishability and Partial Backordering," Management Science 42, 8 (1996), 1093-1104.

Abad, P., "Optimal Price and Lot Size when the Supplier Offers a Temporary Price Reduction over an Interval," Computers and Operations Research 30, 1 (2003), 63-74.

Abad, P. L., "Determining Optimal Selling Price and Lot Size when the Supplier Offers All-Unit Quantity Discounts," Decision Sciences 19, 3 (1988), 622-634.

Boyd, S. P. and L. Vandenberghe, Convex Optimization, Cambridge University Press, 2004. 
Duffin, R. J., E. L. Peterson, and C. Zener, "Geometric Programming: Theory and Application," Wiley, New York, NY, 1967.

Dye, C.-Y., "Joint Pricing and Ordering Policy for a Deteriorating Inventory with Partial Backlogging," Omega 35, 2 (2007), 184-189.

Goh, M. and S. Moosa, "Price Dependent Inventory Model with Discount Offers at Random Times," Production and Operations Management 11, 2 (2002), 139-156.

Grant, M. and S. Boyd, "Cvx Users' Guide for cvx version 1.21,” 2010.

Jørgensen, S. and P. M. Kort, "Optimal Pricing and Inventory Policies: Centralized and Decentralized Decision Making," European Journal of Operational Research 138, 3 (2002), 578-600.

Lee, W. J., "Determining Order Quantity and Selling
Price by Geometric Programming: Optimal Solution, Bounds, and Sensitivity," Decision Sciences 24, 1 (1993), 76-87.

Lim, S., "Solving Robust EOQ Model Using Genetic Algorithm," International Journal of Management Science 13, 1 (2007), 35-53.

Lim, S., "A Robust Pricing/Lot-Sizing Model and Asolution Method Based on Geometric Programming," International Journal of Management Science 14, 2 (2008), 13-23.

Viswanathan, S. and Q. Wang, "Discount Pricing Decisions in Distribution Channels with Price-Sensitive Demand," European Journal of Operational Research 149, 3 (2003), 571-587.

Yu, G., "Robust Economic Order Quantity Models," European Journal of Operational Research 100, 3 (1997), 482-493. 